\title{
Numerical comparison of two boundary meshless methods for water wave problems
}

\author{
Zatianina Razafizana ${ }^{1,2}$, Wen Chen ${ }^{2}$ \& Zhuo-Jia $\mathrm{Fu}^{2}$ \\ ${ }^{1}$ College of Harbor, Coastal and Offshore Engineering, \\ Hohai University, China \\ ${ }^{2}$ Department of Engineering Mechanics, Hohai University, China
}

\begin{abstract}
This paper focuses on numerical comparison on the singular boundary method (SBM) and the method of fundamental solutions (MFS) for water wave problems. These two methods are strong-form meshless boundary collocation methods. These methods are applicable in various problems when the fundamental solution of the governing differential equation is known. To avoid the singularities at origin, the SBM introduces the concept of source intensity factor and circumvents the troublesome placement of the fictitious boundary in the MFS. Numerical results show the efficiency, stability and convergence of the SBM and the MFS through some benchmark examples under two-dimensional semi-infinite harbor problems.

Keywords: singular boundary method, method of fundamental solutions, strong form, harbor wave, collocation method.

\section{Introduction}

A harbor is partially enclosed basin of water connected through one or more opening to the sea. It is a sheltered environment for the mooring of ships and vessels. It is important to understand the interaction between the boundary of harbor and water wave. The scattering of water waves is one of major interesting problems to engineers $[1,2]$. The direct problem of water wave scattering by semi-circular boundary of harbor is our main concern in this paper. Based on assumptions of potential flow and linear wave theory, the problem of water wave scattering can be formulated to $2 \mathrm{D}$ Helmholtz equation.
\end{abstract}


The method of fundamental solutions (MFS) is a meshless technique for the numerical solution of the problems that is approximated by a linear combination of fundamental solution in terms of sources (singularities) which are placed inside the domain of the problem according to Fairweather and Karageorghis [3]. The unknown coefficients of the fundamental solutions are found by forcing the approximation to satisfy the boundary condition according to Karageorghis [4]. The MFS has been used by Fairweather, Karageorghis and Martin for treating the scattering and radiation problems [5].

The singular boundary method (SBM) is a novel meshless boundary methods proposed by $\mathrm{Chen}$ and $\mathrm{Fu}$ [7]. The new numerical method does not require a fictitious boundary to solve problems. The source points can be placed directly on physical boundary. Unlike the MFS, this method uses Inverse Interpolation technique (IIT) to circumvent the singularity of the fundamental solutions at the origin. The method has been applied successfully to interior and exterior Laplace [6-9], elastostatic [10] and Helmholtz [11, 12] problems, which can achieved the accurate and stable numerical results.

\section{Governing equation and numerical method formulation}

\subsection{Governing equation}

Assuming that the water sea bodies are incompressible and non-viscous fluid and the rotational motion is negligible. The governing equation of the water wave is given by

$$
\nabla^{2} \Phi(x, y, z, t)=0, \quad(x, y, z) \in \Omega
$$

where $\nabla, \Omega$ and $\Phi(x, y, z)$ denote the Laplace operator, the domain of interest and the velocity potential, respectively. The boundary conditions are

- Bottom boundary condition

$$
-\Phi_{n}=0, \quad z=-H(x, y) \in \Omega
$$

- Kinematic free-surface boundary condition

$$
-\Phi_{z}=\alpha_{t}-\Phi_{x} \alpha_{x}-\Phi_{y} \alpha_{y}=0, \quad z=\alpha(x, y, t) \in \Omega
$$

- Dynamic free-surface boundary condition

$$
-\Phi_{t}+g z+1 / 2\left(\Phi_{x}^{2}+\Phi_{y}^{2}+\Phi_{z}^{2}\right)=B(t), \quad z=\alpha(x, y, t) \in \Omega
$$

Using the method of separation variable, we have

$$
\Phi(x, y, z, t)=\mathfrak{R} e\left\{u(x, y) f(z) e^{-i \omega t}\right\}
$$

where $f(z)=-i g A / \omega \cosh [k(z+H)] / \cosh (k H)$, in which $g, A, k, \omega$, and $H$ are the acceleration of the gravity, the amplitude of incident wave, the wave number, the wave frequency and the water height, respectively. Substituting 
Eq. (5) into Eq. (1) and using the boundary conditions, we have the Helmholtz equation for water wave:

$$
\left(\nabla^{2}+k^{2}\right) u(x, y)=0, \quad(x, y) \in \Omega
$$

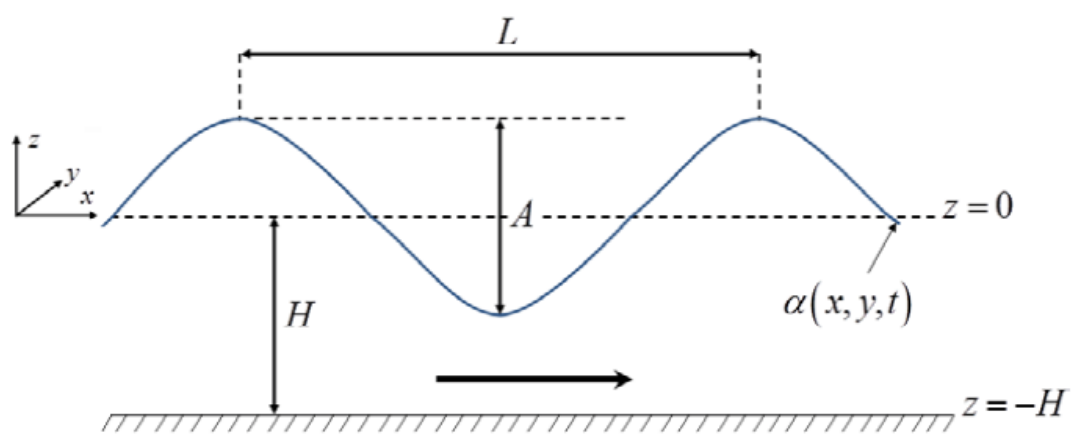

Figure 1: $\quad$ Spatial representation of $\alpha(x, y, t)$.

\subsection{The numerical method formulations}

We consider the Helmholtz equation (6) governing potential problems in two dimensions in semi-infinite domains $\Omega$. Subjected to the boundary conditions

$$
\begin{array}{cc}
u(x)=\bar{u}, & x \in \Gamma_{D} \\
\frac{\partial u(x)}{\partial n}=\bar{q}, & x \in \Gamma_{N}
\end{array}
$$

where $\Delta, u, \Omega, \Gamma_{D}, \Gamma_{N}$, and $n$ are the Laplace operator, potential field, unbounded region in $\mathfrak{R}^{2}$, Dirichlet boundary, Neumann boundary and unit.
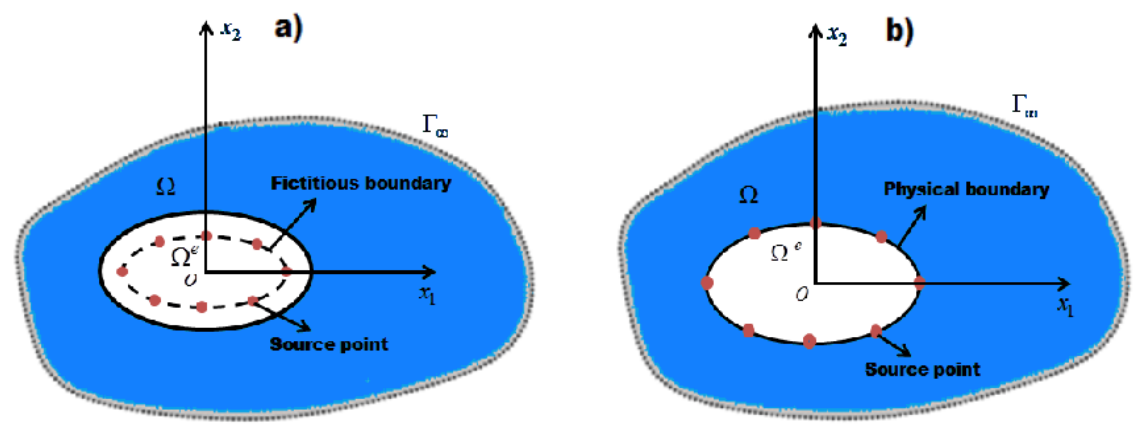

Figure 2: $\quad$ Sketch and node distribution for exterior problems: (a) MFS and (b) SBM. 
The Sommerfeld radiation condition at infinity in 2-D is given by

$$
\lim _{r_{j} \rightarrow \infty} r_{j}^{\frac{1}{2}}\left(\partial / \partial r_{j}-i k\right) u(x)=0
$$

\subsubsection{Method of fundamental solutions (MFS)}

The solution of equations (7)-(9) is approximated by

$$
u\left(x_{m}\right)=\sum_{j=1}^{N} \varepsilon_{j} G\left(x_{m}, s_{j}\right), \quad x_{m} \in \Omega
$$

where $G\left(x_{m}, s_{j}\right)=i H_{0}^{(1)}\left(k r_{m j}\right) / 4$, the Euclidean distance $r_{m j}=\left\|x_{m}-s_{j}\right\|_{2}$ and $i=\sqrt{-1}$. Where $x_{m}, s_{j}, \varepsilon_{j}$, and $G$ represent the $m^{\text {th }}$ collocation point, $j^{\text {th }}$ source point, $j^{\text {th }}$ unknown coefficient to be determined, $H_{n}^{(1)}$ is the $n$th order Hankel function of the first kind.

\subsubsection{Singular boundary method (SBM)}

The singular boundary method formula is presented as

$$
\begin{aligned}
& u\left(x_{m}\right)= \begin{cases}\sum_{j=1}^{N} \alpha_{j} G\left(x_{m}, s_{j}\right), & x_{m} \in \Omega \backslash \Gamma_{D} \\
\sum_{j=1, m \# j}^{N} \alpha_{j} G\left(x_{m}, s_{j}\right)+\alpha_{m} G_{m m}, & x_{m} \in \Gamma_{D}\end{cases} \\
& \frac{\partial u\left(x_{m}\right)}{\partial n_{x}}= \begin{cases}\sum_{j=1}^{N} \alpha_{j} \frac{\partial G\left(x_{m}, s_{j}\right)}{\partial n_{x}}, \quad x_{m} \in \Omega \backslash \Gamma_{N} \\
\sum_{j=1, m \# j}^{N} \alpha_{j} \frac{\partial G\left(x_{m}, s_{j}\right)}{\partial n_{x}}+\alpha_{m} \overline{G_{m m}}, & x_{m} \in \Gamma_{N}\end{cases}
\end{aligned}
$$

where $\alpha_{j}, G_{m m}, \overline{G_{m m}}, n_{x}$, and $N$ represent the $j^{\text {th }}$ unknown coefficient to be determined by the imposing the boundary condition, and the source intensity factors, the outward normal unit on the collocation points $x_{m}$, and the number of source points $s_{j}$, respectively. The source intensity factors are determined by the Inverse Interpolation Technique (IIT).

\subsubsection{Inverse interpolation technique (IIT)}

This section introduces a simple numerical technique, called inverse interpolation technique (IIT) $[6,7]$, to calculate the source intensity factors for Laplace equation. Then, we use the relation between the Laplace and Helmholtz fundamental solutions to determine the source intensity factors for 
Helmholtz equation [13]. By using the interpolation formula (11), the influence coefficients $\beta_{j}$ and $\overline{\beta_{j}}$, are given by the following relation:

$$
\begin{gathered}
\left\{G_{0}\left(y_{k}, s_{j}\right)\right\}\left\{\beta_{j}\right\}=\left\{u_{I}\left(y_{k}\right)\right\} \\
\left\{\frac{\partial G_{0}\left(y_{k}, s_{j}\right)}{\partial n_{x}}\right\}\left\{\overline{\beta_{j}}\right\}=\left\{u_{I}\left(y_{k}\right)\right\}
\end{gathered}
$$

Replacing the sample points $y_{k}$ with the boundary collocation points $x_{m}$, the source intensity factors for Laplace equation can be calculated by:

$$
\begin{gathered}
G_{m 0}=\left[u_{I}\left(x_{m}\right)-\sum_{j=1, x_{m} \# s_{j}}^{N} \alpha_{j} G_{0}\left(x_{m}, s_{j}\right)\right] / \beta_{j}, x_{m}=s_{j}, x_{m} \in \Gamma_{D} \\
\overline{G_{m 0}}=\left[\frac{\partial u_{I}\left(x_{m}\right)}{\partial n}-\sum_{j=1, x_{m} \# s_{j}}^{N} \alpha_{j} \frac{\partial G_{0}\left(x_{m}, s_{j}\right)}{\partial n}\right] / \overline{\beta_{j}}, x_{m}=s_{j}, x_{m} \in \Gamma_{N}
\end{gathered}
$$

The source intensity factors for Helmholtz problem can be written as

$$
\begin{aligned}
& G_{m m}=G_{m 0}-1 / 2 \pi(\ln (k / 2)+\gamma-i \pi / 2), x_{m}=s_{j}, \quad x_{m} \in \Gamma_{D} \\
& \overline{G_{m m}}=\overline{G_{m 0}}, \quad x_{m}=s_{j}, \quad x_{m} \in \Gamma_{D}
\end{aligned}
$$

\section{Numerical results}

The efficiency, accuracy, stability and convergence are tested by calculating the relative error (Rerr):

$$
\text { Rerr }=\sqrt{\sum_{i=1}^{N T}|u(i)-\bar{u}(i) / \bar{u}(i)|^{2} / N T}
$$

where $u$ and $\bar{u}$ are the numerical solution and the exact solution of the total field. Here the number of the tested points $N T$ is 808 , which uniformly distributed inside the semi-cirque with inner radius 1 and outer radius 8 .

Example 1: Scattering of water wave problems in the harbor. The problem is semi-infinite.

We consider incident wave $u_{i}(x)=e^{i k x}$, and reflected wave $u_{r}(x)=e^{-i k x}$ scattered by soft and hard semi-circular as showing in Fig. 2 . The total field of scattering is $u=u_{s}+u_{i}+u_{r}$, which satisfies the Helmholtz equation (6). The boundary conditions are given by 
- Rigid boundary

$$
\frac{\partial u_{s}(x)}{\partial n}+\frac{\partial u_{i}(x)}{\partial n}+\frac{\partial u_{r}(x)}{\partial n}=0, \quad x \in \partial \Omega
$$

- Soft boundary

$$
u_{s}(x)+u_{i}(x)+u_{r}(x)=0, \quad x \in \partial \Omega
$$

The analytical solution of scattering wave is written as [14].

- Scattering wave for hard boundary

$$
\begin{aligned}
& u_{s}(r, \theta)=-2 \frac{J_{0}^{(1)}(k p)}{H_{0}^{(1)}(k p)} H_{0}^{(1)}(k r) \\
& -2 \sum_{t=1}^{\infty}\left(i^{t}+(-i)^{t}\right) \frac{-k p J_{t-1}^{(1)}(k p)+t J_{t}(k p)}{k p H_{t-1}^{(1)}(k p)-t H_{t}^{(1)}(k p)} H_{t}^{(1)}(k r) \cos (t \theta)
\end{aligned}
$$

\section{- Scattering wave for soft boundary}

$$
\begin{aligned}
& u_{s}(r, \theta)=-2 \frac{J_{0}^{(1)}(k p)}{H_{0}^{(1)}(k p)} H_{0}^{(1)}(k r) \\
& -2 \sum_{t=1}^{\infty}\left[i^{t}+(-i)^{t}\right] \frac{J_{t}^{(1)}(k p)}{H_{t}^{(1)}(k p)} H_{t}^{(1)}(k r) \cos (t \theta)
\end{aligned}
$$

where $H_{t}^{(1)}, J_{t}^{(1)},(r, \theta), k$, and $p$ represent the $t$-th order of Hankel function the first kind, the $n$th order of Bessel function the first kind, polar coordinates of the domain point, the wave number and the radii of the physical domain, respectively.

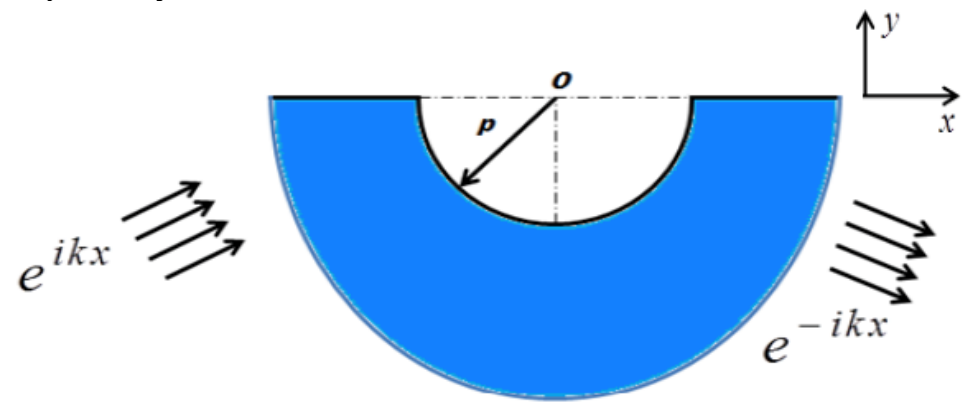

Figure 3: Sketch of water wave scattering in the harbor.

Figure 4 shows the error analysis of the SBM and MFS for 2D scattering of water wave by soft (a) and hard (b) semi-circular harbour boundaries. To test the stability of MFS, we took two different fictitious radiuses. The fictitious radiuses 

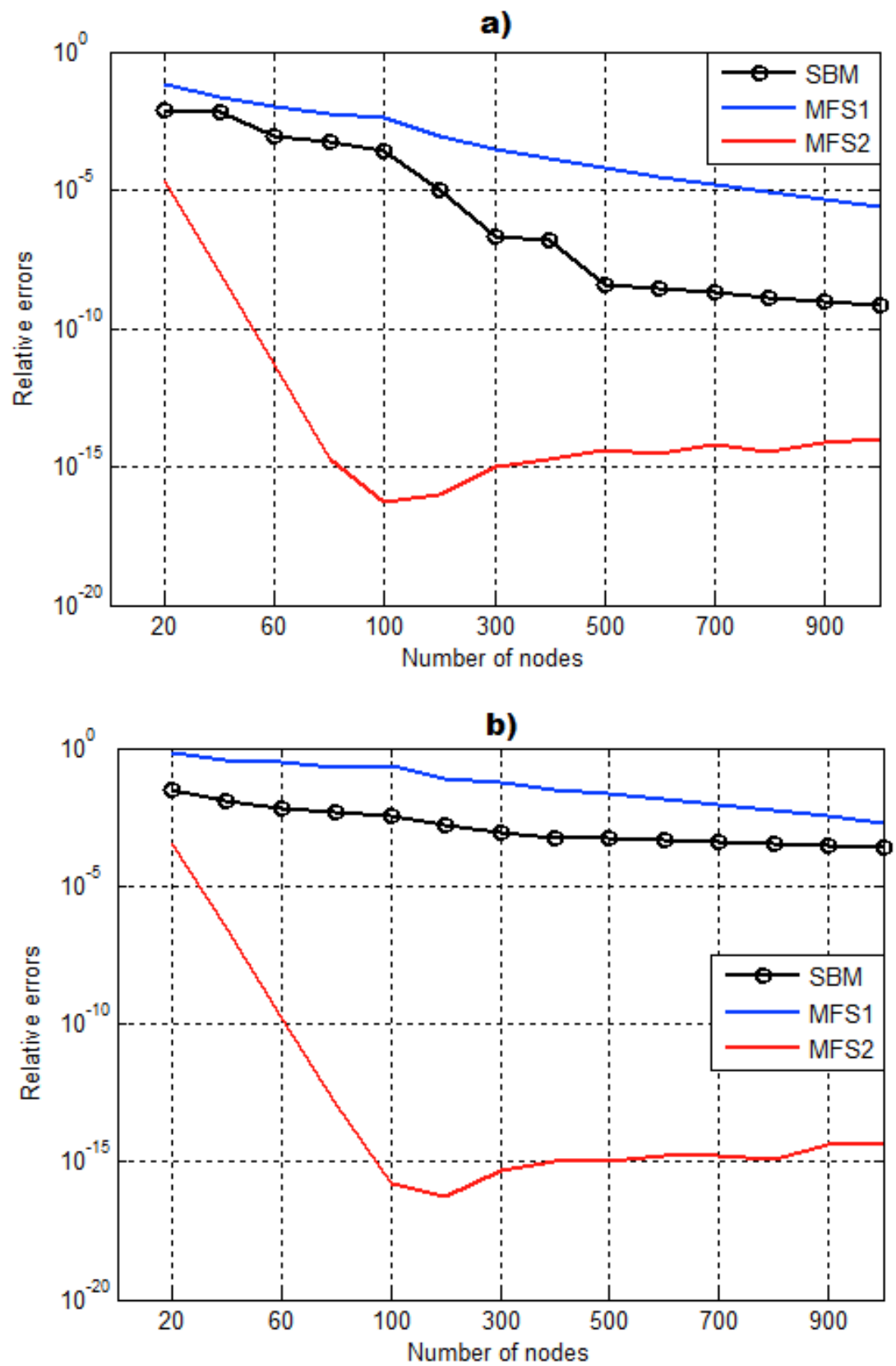

Figure 4: Numerical results of example for various numbers of nodes: (a) soft boundary and (b) rigid boundary. 
are 0.99 for MFS1 and 0.7 for MFS2. From Figure 4, we can observe that MFS is not stable with different fictitious radiuses. Among these results, the MFS2 has the most accurate numerical solutions but the MFS1 has the worst numerical solutions. While the SBM avoids the controversial fictitious boundary in MFS, and provides the stable and accurate results.

\section{Conclusions}

This paper presents the comparison numerical on the singular boundary method (SBM) and method of fundamental solutions (MFS) for harbor problems. The MFS and SBM are boundary meshless collocation methods. Both of them use the fundamental solution of the governing differential equation as basis function to avoid singular numerical integrals in the boundary element method. In addition, the SBM introduces the source intensity factors to eliminate the singularities of fundamental solutions at origin and avoid the fictitious boundary in the MFS. Numerical results verify that the SBM is more stable than the MFS.

\section{References}

[1] J. T. Chen, Y. T. Lee, Y. J. Lin (2009): Interaction of water wave with vertical cylinders using the null-field integral equations. Applied Ocean Research, vol. 31, pp. 101-110.

[2] J. T. Chen, Y. J. Lin, Y. T. Lee, C. F. Wu (2011): Water wave interaction with surface-piercing porous cylinders using the null-field integral equations. Ocean Engineering, vol. 38, pp. 409-418.

[3] G. Fairweather, A. Karageorghis (1998): The method of fundamental solutions for elliptic boundary value problems. Advances in Computational Mathematics, pp. 69-95.

[4] A. Karageorghis (2001): The method of fundamental solutions for calculation of the Eigen values of Helmholtz Equation. Applied Mathematics Letters, pp. 837-842.

[5] G. Fairweather, A. Karageorghis and P. A. Martin (2003): The method fundamental solution for scattering and radiation problems. Engineering Analysis with Boundary Elements, vol. 27, pp. 759-769.

[6] W. Chen, F. Z. Wang (2010): A method of fundamental solutions without fictitious boundary. Engineering Analysis with Boundary Elements, vol. 34, pp. 530-532.

[7] W. Chen, Z. J. Fu (2010): A novel numerical method for infinite domain potential problems. Chinese Sciences Bulletin, vol. 55, pp. 1598-1603.

[8] Y. Gu, W. Chen, and X. Q. He (2012): Singular boundary method for steady-state heat conduction in three dimensional general anisotropic media. International Journal of Heat and Mass Transfer, vol. 55, pp. 48374848 .

[9] W. Chen, Z. J. Fu and Xing Wei (2009): Potential Problems by Singular Boundary Method Satisfying Moment Condition. Computer Modeling in Engineering and Sciences, vol. 54, pp. 65-85. 
[10] Y. Gu, W. Chen, and C. Z. Zhang (2011): Singular boundary methods for solving plane strain elastostatic problems. International Journal of Solids and Structures, vol. 48, pp. 2549-2556.

[11] Z. J. Fu, W. Chen (2010): A novel boundary meshless method for radiation and scattering problems. In: C.-Z. Zhang. M. H. Aliabadi, M. Schanz, editor. Advances in Boundary Element Techniques XI. Berlin Germany: EC Ltd, United Kingdom; pp. 83-90.

[12] Z. J. Fu, W. Chen, C. S. Chen (2012): Singular boundary method for radiation and wave scattering: Numerical aspects and applications. Proc. $23^{\text {rd }}$ International Congress of Theoretical and Applied Mechanics (ICTAM 2012).

[13] Y. Gu, W. Chen, and J. Zhang (2012): Investigation on near-boundary solutions by singular boundary methods. Engineering Analysis with Boundary Elements, vol. 36, pp. 1173-1182.

[14] D.H. Tsaur and K.-H. Chang (2009): Scattering of SH waves by truncated Semicircular Canyon. Journal of Engineering Mechanics, vol. 135, pp. 862870 . 